\title{
Configuring a system for hydraulic oil contamination management
}

\author{
Petru Dusa*, Eugen Purice, Radu Lupascu, Iustina Ripanu and Gabriel Fandarac \\ Department of Machine Manufacturing Technology, Gheorghe Asachi Technical University, \\ Mangeron, 59A, 700050, Iasi, Romania
}

\begin{abstract}
Through contamination, we can understand anything foreign to a hydraulic system. In this paper we only refer to particle contamination. Analyzing and optimizing processes with regard to component cleanliness and fluid purity is the target of contamination management. The paper proposes a conceptual installation solution for the use of particle counters in order to optimize the measurement process. There is also a way of using the facility within a contamination management system.
\end{abstract}

\section{Introduction}

Contamination refers anything foreign in the hydraulic system. There are at least two types of contaminants: particulate and chemical. Particulate contaminants include dirt, sand, metal or rubber wear particles. Chemical contaminants include heat, water and air.

Contamination control means preventing contaminants from entering into hydraulic system and placing filters in adequate locations to stop contaminants to find a way into the fluid. Analysis and optimization of processes with regard to the cleanliness of components and the purity of the fluids is the target of contamination management $[1,2]$. During the time sophisticated instruments and methods for the hydraulic fluid's cleanliness assessment was developed [3-14] and the industry is aware by the increase in the numbers of particles reported compared to the distribution based on optical counters. Belong 1960-1980 National Aerospace Standard NAS 1638 [13] was a particulate contamination coding system to convert particle count at various size ranges into classes. Become from 1987 ISO4406 [10] brings a new method for coding level of contamination by solid particles. Contamination standards for automatic particle counter calibration also changed.

\section{Problem definitions}

One problem is that old and new data from automatic particle counters, as a knowledge base, need correlation. The old data from automatic particle counters served for manufacturing companies in the development management.

Another problem is that the contamination measurements, especially when using automatic particle counters, require special washes after each measurement. To perform

${ }^{*}$ Corresponding author: pdusa@,tcm.tuiasi.ro 
these washes, it is usually used separate washing installations which cause disconnection of the counters from the investigated circuit and connection to the washing facilities. This approach results in time consuming, and connections and disconnections can become sources of contamination.

This study is carried out by analyzing standards and instruments correlation concerning hydraulic oil contamination with the target to configure a system for hydraulic fluid contamination management to serve hydraulically operated machines. More precisely the configured system for hydraulic oil contamination management needs to assure guidelines for setting target cleanliness level, contamination control and designing filtration system by correlation of old data (existent knowledge) with new oil cleanliness systems requirements.

\section{Conceptual solution and research method}

To decrease the number of connections / disconnections during measurement was designed a facility that allows, on the one hand, perform measurements of contamination and on the other hand flushes counter and prepare for another measurement. A view of the installation is shown in Figure 1, and the principle scheme is shown in Figure 2 (on Figure 1 and 2 the items has the same reference numbers). The installation comprises a hydraulic pump 1 driven by an electric motor, the circuit being provided with a pressure relief valve 2 . The flow coming from the pump may be directed through a three-way valve 3 by the two circuits. A circuit continues through a filter 4, a pressure gauge 5, a restrictor 7 and the hose 8 closes the circuit to the oil tank 13. A quick coupling connection unit 6 allows connection to automatic particle counter unit B. This circuit can be considered the washing circuit for the automatic particle counter unit B. A second circuit proceeds from a three-way valve 3 via path 2 through the restrictor 10 and closes through the flexible hose 11 to the oil reservoir 13. A quick coupler 12 allows connection to automatic particle counter unit B and this second circuit is a measurement circuit for contaminated oil. The two circuits are selected alternatively as required.

Restrictors 7 and 10 are designed to provide the required pressure for measurements. The tank 13 is even the sample of oil harvested in accordance with the standards. As can be seen, the tank 13 is changeable and changes with each sample.

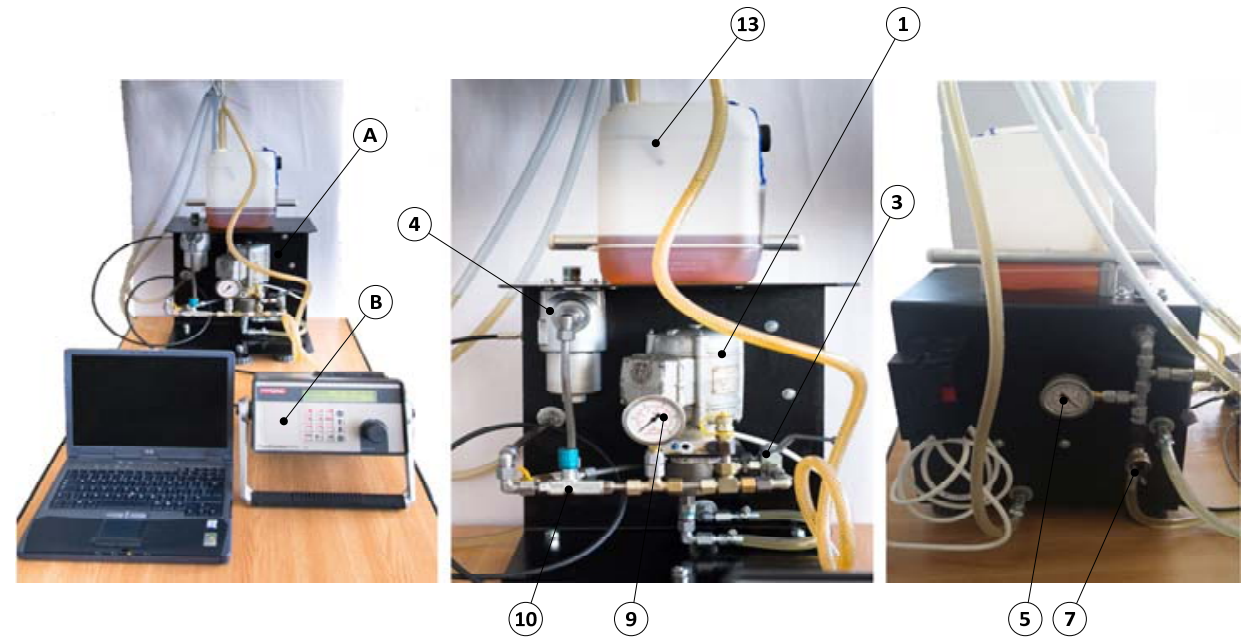

Fig. 1. View of the installation with two circuits (measurement and flushes).

The installation, identified as a subassembly A, is completed with a computer running an interfacing application with an automated particle counter identified by B. 
The methodological approach concerns in using for the measurement of the same oil sample the both ISO4407 [11] and ISO 11500 [12] coding system standards.

This means that for the same sample, two methods of contamination assessment will be used: the determination of particulate contamination by the counting method using an optical microscope and the determination of particulate contamination by automatic counting using the light extinction principle.

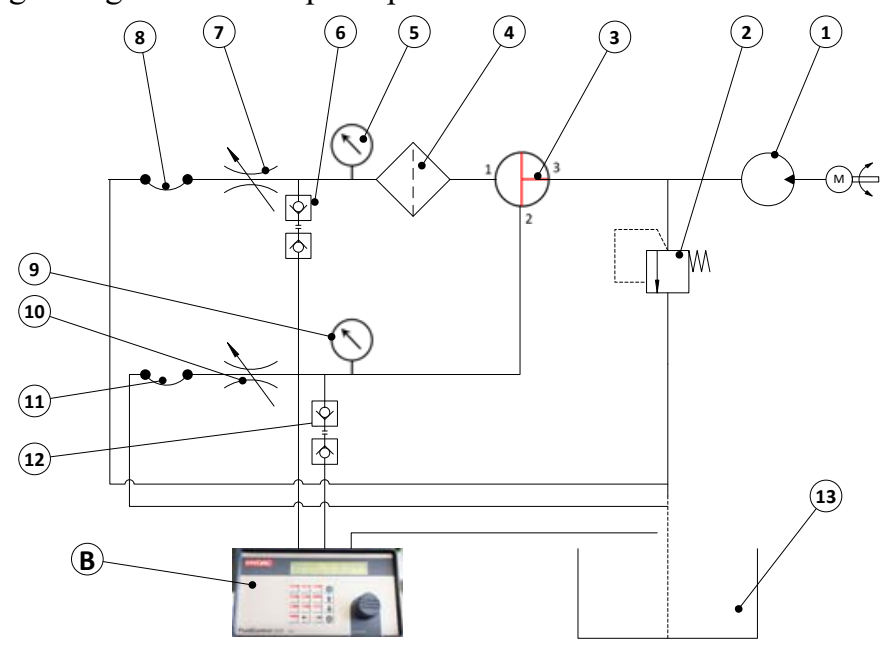

Fig. 2. Principle of the installation with two circuits (measurement and flushes).

As instrument we used an automatic particle counters Hydac FluidControl Unit FCU 2000 series, fiber-optic infrared sensor technology for detecting solid contaminants in highpressure lines in hydraulic systems and for bottle sampling and tank analysis which principle is presented on Figure 3.

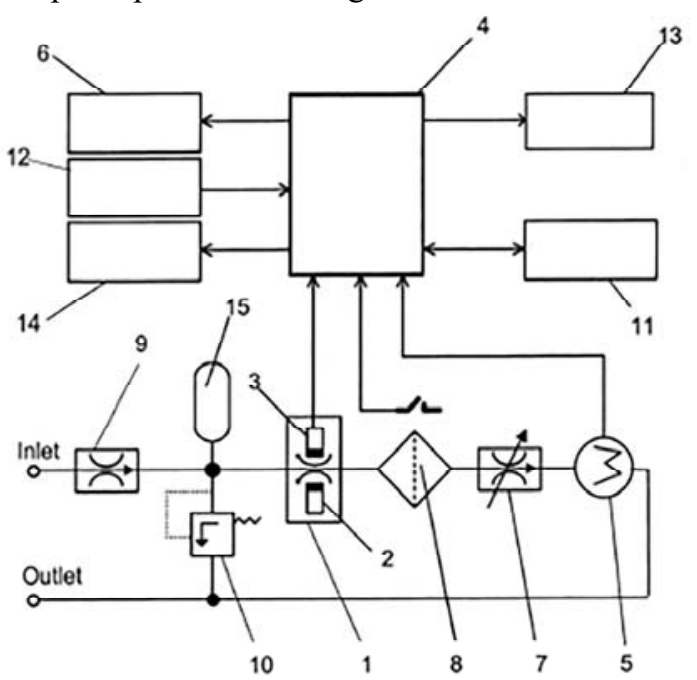

Legend:

\begin{tabular}{|c|l|}
\hline Item & \multicolumn{1}{|c|}{ Designation } \\
\hline 1 & Optical sensor \\
\hline 2 & Infrared LED \\
\hline 3 & Photodetector \\
\hline 4 & Evaluation electronics \\
\hline 5 & Flow rate sensor \\
\hline 6 & Display \\
\hline 7 & Flow rate control valve (adjustable) \\
\hline 8 & Fitter \\
\hline 9 & Flow control valve \\
\hline 10 & Pressure control valve \\
\hline 11 & Serial interface \\
\hline 12 & Keyboard \\
\hline 13 & Relay \\
\hline 14 & Printer \\
\hline 15 & Pressure accumulator \\
\hline
\end{tabular}

Fig. 3. Principle schema of automatic particle counters (FluidControl Unit FCU 2000) [6].

A validation of the both results is made using determination of particulate contamination by the counting method using an optical microscope [10]. The reference photographs (Figure 4) enable a quick evaluation of fluid contamination (cleanliness class rating). A microscope is used to determine the type of particle. 


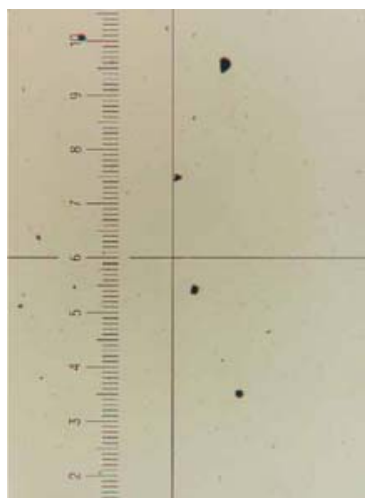

a.

Comparison photograph for fluid contamination class NAS $1638 \quad$ Class 7 ISO 4406: Class 18/16/13 SAE AS 4059 Class 8 Magnification: x100 1 Scale $\operatorname{mark}=\mathbf{1 0} \boldsymbol{\mu m}$

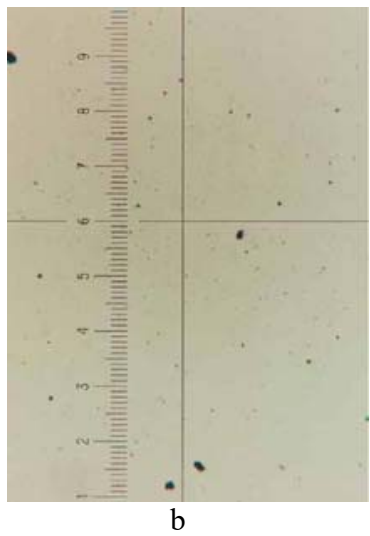

Comparison photograph for fluid contamination class NAS 1638 Class 8 ISO4406: Class 19/17/14 SAE AS4059 Class 9 Magnification: x100 1 Scale $\operatorname{mark}=\mathbf{1 0} \boldsymbol{\mu m}$

Fig. 4. Samples for comparison of results by counting method using an optical microscope [11].

\section{Research results}

Using the installation and the method presented, measurements were made on several oil samples. Oil samples were taken from hydrostatic-driven construction equipment. Since the interest of the research was to establish correlations between the old data held by organizations (knowledge as an asset) and the new coding systems, attention was focused on oils whose contamination was in classes 7;8 (NAS 1638) respectively 18/16/13 ; 19/17/14 (ISO 4406).

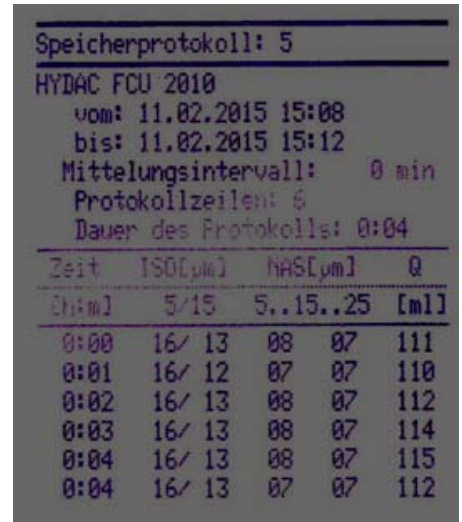

a.

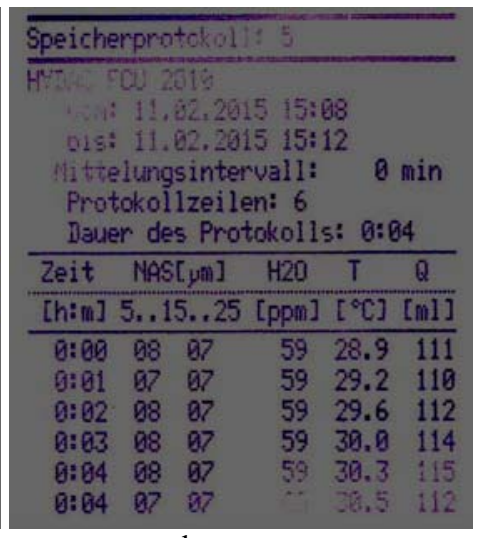

b.

Fig. 5. Examples of measurements results from automatic particle counter.

Figure 5 shows an example of a measurement result using an automated particle counter according to ISO 11500 [12]. 


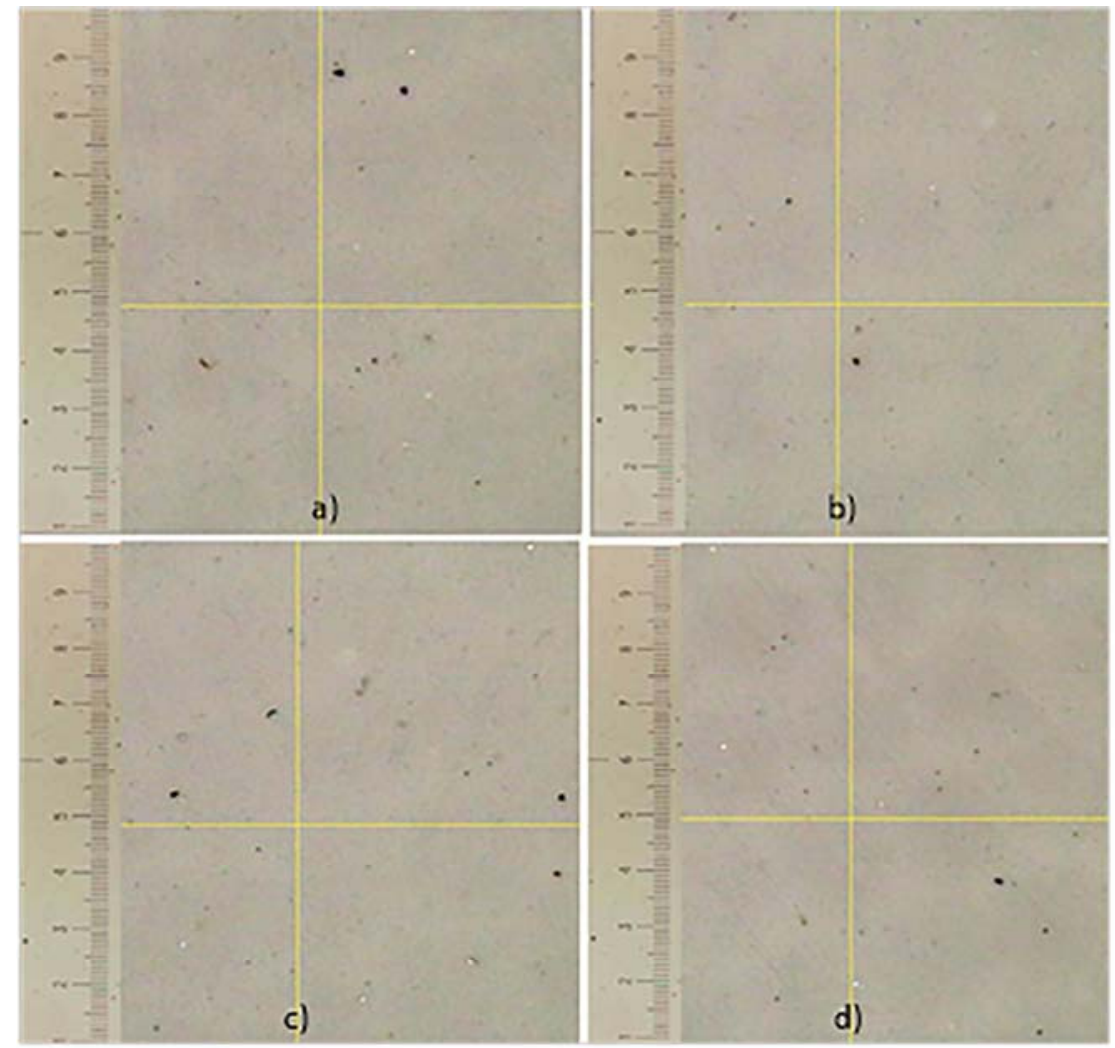

Fig. 6. Examples of measurement results used for comparison with samples by counting method using an optical microscope.

Figure 6 shows examples of results obtained by measurement using an optical microscope according to ISO 4407 [11].

According to the standard, the entire membrane is investigated to confirm a good distribution of the particles. Counter patterns are also set up (50 squares, 20 squares, and 10 squares). The 20-square pattern was used and Figure 6 shows the 9 (a), 10 (b), 11 (c), 12 (d) squares according to the ISO 4407 standard [11].

\section{Discussions}

As shown in Figure 5 the automated particle counter draws reports on oil contamination. Reports present the timing of the assessment, ISO classification, NAS classification, and the amount of liquid being evaluated. In another configuration (as shown in Figure 5b), the reports present the time of evaluation, the NAS class, the amount of water expressed in particles per minute, the oil temperature at the time of the investigation, and the amount of liquid being evaluated.

Figure 6 shows the results of investigating the same oil sample by counting method using an optical microscope. This means that the particles should be divided into size classes, evaluated the number of particles in each class, and the results should be presented in the form of the number of particles in each class.

Another approach to qualifying contamination of oils is the use of comparison photograph for fluid contamination class [5]. This is the method we used and therefore the results obtained and shown in Figure 6 were compared with the samples of Figure 4. 
The photos of Figure 6 were compared with the whole set of comparative photographs [5]. The most consistent resemblances have been highlighted for comparison photographs of NAS 7 and 8 classes (as shown in Figure 4).

As can also be seen from the measurements shown in Figure 5 (developed using an automated particle counter) the investigated oil contamination classes are NAS 8 (for particles 5-15 microns) and NAS 7 (for particles 15-25 microns) corresponding to the ISO $18 / 16 / 13$ class.

\section{Conclusions}

It can be said that the configured solution consisting of a two-circuit system (measurement and washing) provides efficient possibilities of oil contamination management by reducing the number of connections / disconnections, and the tank of the installation is made up of the sampling container.

Organizations have long developed a knowledge base on the recommended levels of cleanliness for different applications. The diversity of hydraulic systems in terms of pressure, environment, lubrication etc. can be a source of conflict when comparing levels of contamination. A contamination management system requires at least two methods to assess the level of contamination.

\section{References}

1. M. Singh G.S. Lathkar S. K. Basu, Failure Prevention of Hydraulic System Based on Oil Contamination, J. Inst. Eng. India Ser. C (July-September 2012) 93(3):269-274

2 Caterpillar Hydraulic System Management Guide, available at: https://parts.cat.com/wcsstatic/pdfs/PEGP6028-05.pdf

3. FILTRATION IN BRIEF, available at: http://www.ufihyd.com/pdf/en/filtrazione e.pdf

4. HYDAC FILTER SYSTEMS GMBH Practical Contamination Management From Processing to Delivery, E 7.604.1/05.09, available at: http://www.hydac.com

5. HYDAC FILTERTECHNIK GMBH Contamination handbook, Prospekt-Nr. 7.603.3/07.06, available at: http://www.hydac.com

6. HYDAC FILTERTECHNIK GMBH FCU 2210-5 FluidControl Unit Operating and Maintenance Instructions, available at: http://www.hydac.com

7. Donaldson, FLUID CLEANLINESS comparison guide, available at: https://www.donaldson.com/en/ih/support/datalibrary/059799.pdf

8. Ernie Parker, Hydraulic Fluid Contamination and Assessment, available at: http://insidepenton.com/machinedesign/HydraulicFluidContaminationandAssessment.pdf

9. A Guide to Contamination Control for Hydraulic and Lubrication Systems, available at: https://www.parker.com/literature/HydraulicFilterDivisionEurope/fdhb138g1.pdf

10. ISO 4406 Hydraulic fluid power - Fluids - Method for coding the level of contamination by solid particles

11. ISO4407 Hydraulic fluid power - Fluid contamination - Determination of particulate contamination by the counting method using an optical microscope

12. ISO11500 Hydraulic fluid power - Determination of particulate contamination by automatic counting using the light extinction principle

13. NAS 1638 Cleanliness Requirements of Parts Used in Hydraulic Systems

14. SAE AS 4059 Aerospace Fluid Power - Cleanliness Classification for Hydraulic Fluids 